\title{
RAPID PROTOTYPING OF DISTRIBUTED MECHATRONIC APPLICATIONS
}

\author{
Markus Deppe, Carsten Homburg
}

This paper presents a unified approach to optimize the information-processing structure of a distributed mechatronic system with respect to the inherent physical structure. It is based on a process-oriented model representation by means of Mechatronic Processing Objects. Hierarchical clustering mechanisms allow the treatment of parallel and distributed system architectures. The possibilities of employing a specialized dataflow processor for the rapid prototyping of distributed mechatronic applications are discussed. As an example, the design of an active vehicle suspension is presented.

\section{Introduction}

Mechatronic systems are complex, modern technical systems comprising mechanical, hydraulic, and electric parts that are integrated by means of information processing in order to convey optimal functionality. A typical mechatronic system consists of hierarchically organized functional units with control structures on each level.

The design process of a mechatronic system typically starts with a topological, hierarchically organized model of the system, describing the significant phenomena on a sufficiently abstract level. Based on this hierarchical, physical model of the mechatronic system, a hierarchical, partitioned information-processing structure has to be found which reflects the inherent physical structure of the system and meets real-time requirements. As a result of this process of restructuring the model and designing the information structure, the mechatronic engineer obtains a distributed model of the mechatronic system, including model partitioning, task allocation, and communication structure. Distributed hardware-in-the-loop simulations of this parallel model on a rapid prototyping hardware allow a fast and flexible validation of the control and the communication structure. The requirements and structure of the controller network in the final mechatronic product can be directly derived from this parallel model.

The approach presented is based on a process-oriented model representation by means of Mechatronic Processing Objects. A Mechatronic Processing Object (MPO) is a uniform model representation active at all stages from modeling to controller realization, perfectly suited for off-line and on-line numerical evaluation of the 
behavior of the system. Hierarchical clustering mechanisms allow the treatment of parallel and distributed system architectures. Software tools for manual or automatic model partitioning and restructuring, code generation, distributed off-line simulation, and hardware-in-the-loop simulation can work on this process-oriented model representation efficiently at short turn-around times.

Since the behavior of the system on the process-oriented representation level is described as a calculation graph, a dataflow architecture is an ideal hardware platform for the rapid prototyping of mechatronic information-processing structures. The possibilities of employing a specialized dataflow processor for the rapid prototyping of distributed mechatronic applications will be discussed in the following.

Finally, the approach presented is applied step by step to the informationprocessing design of an active car suspension with a distributed and hierarchical controller structure and a test implementation on a transputer network.

\section{Mechatronic Processing Objects}

In order to simplify modeling and to keep it apart from numerical processing, three different levels of model description languages for mechatronic systems were defined at MLaP (Hahn and Richert, 1994): O-DSS (Objective-Dynamic System Structure) on the structural level to specify the system topology by descriptions proper to the various technical disciplines, DSL (Dynamic System Language) to represent system dynamics on the mathematical level, and DSC (Dynamic System Code) as a compact, process-oriented, yet hardware-independent description of the system behavior. Each of these descriptions can automatically be transferred to the lower levels.

The efficient design and evaluation of a distributed, mechatronic informationprocessing structure requires very flexible facilities for model partitioning and scheduling of communication, along with short turn-around times for the derivation and download of the distributed model. Additionally, hardware-in-the-loop simulations require fast code to realize cycle rates of $1 \mathrm{kHz}$ and above. To meet these demands, the Mechatronic Processing Objects were introduced at MLaP (Homburg, 1998).

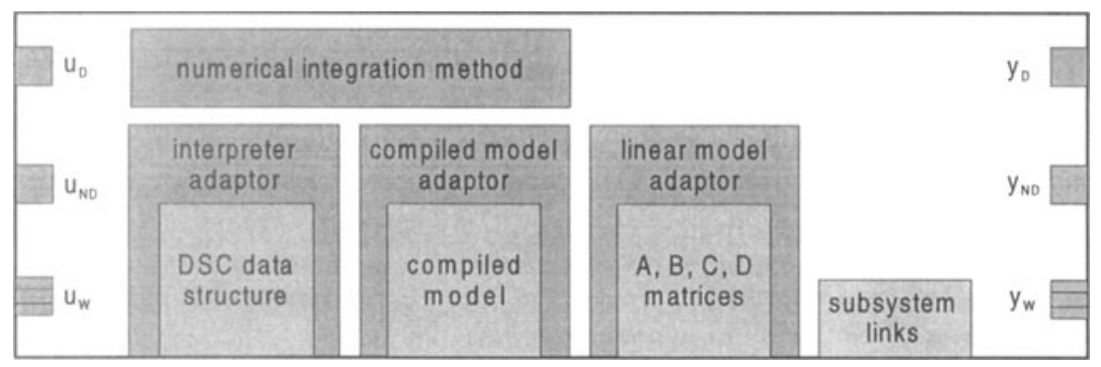

Figure 1. Mechatronic Processing Object (MPO).

A Mechatronic Processing Object (MPO) is a modular model representation on the process-oriented level based on DSC. It is an executable instance of a particular 
model component active at all design stages from modeling up to controller realization. The schematic architecture of a MPO is shown in Figure 1. An MPO contains the directed input-output behavior of a mechatronic system component in form of an interpretable DSC data structure, compiled machine code, or a linear matrix representation. All of these representations can be loaded and exchanged dynamically. Access to the different model representations is encapsulated in specific adaptors that can also be loaded dynamically. This allows a lean implementation that is especially necessary for hardware-in-the-loop simulations. Multiple MPOs can be interlinked to make up a complex system, and the different model representations can be mixed.

A special feature of an MPO is its ability to adapt the order of evaluating the inner equations to the coupling context; this is even possible with previously compiled model components. One system type has to be compiled just once and can then be used in any coupling context. The time-consuming task of recompilation after each modification of the coupling structure is no longer necessary. This is achieved by dividing the model code into three code classes in conjunction with black-box and white-box communication described in the next section. Regarding the input-output dependencies, the code to calculate the behavior of the system can be classified as follows (Schröer, 1994):

- Indirect link code (ND code). This code contains the computations of the output quantities $y_{N D}$ that depend exclusively on the current state quantities. An immediate computation of this code is possible because no current input values are required. Multiple MPOs can execute this code class in parallel.

- Direct link code ( $D$ code). This code contains the computations of output quantities $y_{D}$ that directly depend on the current values of the input quantities $u_{D}$. The current input values $u_{D}$ must be available before computation of this code class can start. Multiple MPOs have to execute this code class sequentially.

- State code ( $S$ code). This code contains the computation of the state derivatives $d x / d t$ and the new system state by numerical integration. The current values of all input quantities $u_{D}$ and $u_{N D}$ must be available. Multiple MPOs can again execute this code class in parallel.

For distributed, coupled MPOs a correct evaluation order of the direct link code is computed throughout the network in an initialization phase. Dynamic computation of the evaluation order at runtime is also possible.

The modular architecture of an MPO guarantees high portability of the concept presented. Each MPO as a stand-alone, active program object can be implemented by a co-routine, a thread, a process, or a process network. Minimum requirement is an ANSI-C compiler; the non-preemptive co-routine solution does not need any support by the operating system.

\section{Distributed Information Processing}

In order to structure the information processing of a mechatronic system, three levels of mechatronic systems were introduced at MLaP (Honekamp et. al., 1997). The 
lowest level is represented by Mechatronic Function Modules (MFMs), which encapsulate mechanical, hydraulic, or electric system components with embedded local control, each performing a very specific task. On the next level, individual Mechatronic Function Modules are coupled to make up an Autonomous Mechatronic System (AMS). On this level, there are mainly two different kinds of couplings. First of all, you have mechanical couplings resulting from the MFMs interacting and the passive mechanical frame of the system. Additionally, there are many informationprocessing interconnections to convey optimum functionality. Cross-linked Mechatronic Systems (CMS) represent the top level of this hierarchy. They are made up of groups of AMSs, coupled exclusively via exchange of information data.

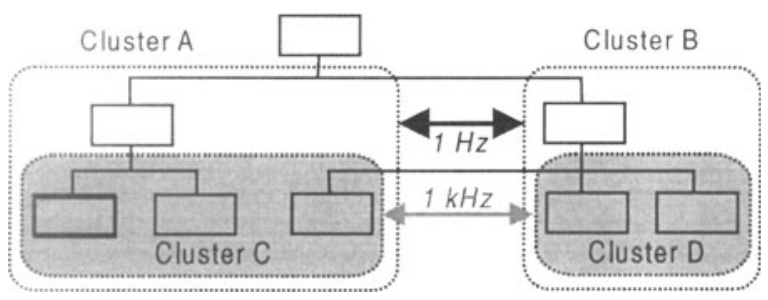

Figure 2. Structuring of the distributed information processing with clusters.

To support hierarchical, distributed information-processing structures, MPOs can be grouped to form hierarchical clusters (Figure 2). A cluster is a processing unit containing a part of the model. It can be mapped to a thread, process, processor, or even a processor network. Clusters can be hierarchically organized with possibly different communication rates on each level. In early design stages, the hierarchical model of the physical structure of the system, specified by the mechatronic engineer, typically does not map to an optimal distributed information-processing structure. The cluster concept provides additional structuring mechanisms to organize the entire system in a very efficient way as regards communication aspects and timing restrictions. In order to optimize the information-processing structure of the system and to find an efficient arrangement of MFMs, AMSs, and CMSs, the origina hierarchical model of the physical structure of the system has to be restructured tr map the cluster structure. Adaption of the physical model to a given cluster structur: can be executed automatically by means of software tools.

Hierarchical communication at possibly different rates on each level require special consideration. MPOs provide two types of communication: The black-bo communication contains two input operations per cluster and cycle for $u_{D}$ and $u_{N D}$ a well as two output operations for $y_{N D}$ and $y_{D}$. This type of communication forces th MPO to act like a black box that reads inputs and writes outputs in two large step: Intermediate input or output operations of input or output values that are already vali are not possible. With increasing cluster size, exclusive use of black-box communi cation will result in unnecessary deadlocks because of algebraic loops. In order t solve this problem, an MPO provides a mechanism for white-box communication This mechanism allows multiple, partial input and output operations of $u_{D}$ and $y_{D}$ vi. the white-box communication variables $u_{W}$ and $y_{\mathrm{w}}$. The scheduling of input and outp operations can be mastered on subcluster levels across cluster boundaries. This allov calculation of a valid evaluation order according to the coupling context $\mathrm{nn}$ 
cluster level. High-level communication at typically lower communication rates is performed by black-box communication. White-box communication is especially necessary for the processing of high-frequency mechanical interactions caused by the mechanical frame of a mechatronic system. These interactions are typically handled on the lower cluster levels.

Optimization of the information-processing structure is supported by several tools. Given a specific processor network and a fixed communication rate, the DSC-based LoDiT (Homburg and Naumann, 1996) calculates a flat cluster structure with nearly optimal speedup. The result can be used as a basis for further manual structure optimizations. For the analysis of real-time properties, CHaRy (Altenbernd, 1996) can be used. It provides an input interface for DSC model descriptions. Visualization tools present partitioning results, schedules, and code dependencies according to the engineer's needs.

\section{Rapid Prototyping with Dataflow Architectures}

Rapid prototyping of a mechatronic system is impossible without hardware-in-theloop simulations. The design of the distributed control and information-processing structure has to be evaluated with regard to real mechanical, hydraulic, or electric components. A suitable hardware has to provide a flexible and scalable network of processors. The simulation of complicated mechanical components with their interactions via the passive mechanical frame requires high computation and communication rates of the same order. High data throughput must be provided for the connection to the physical process. Additionally, the processing of floating-point values is necessary because in early hardware-in-the-loop simulations the engineer does not possess detailed information on the ranges of each system variable.

Dataflow processors are a promising rapid-prototyping hardware architecture that meets these requirements. A dataflow graph can be directly derived from the DSC model representation of a MPO and mapped to a dataflow processor, exploiting the inherent parallelism of the model. Dataflow subgraphs can be interpreted as parallel model partitions, representing MPO clusters. Connectivity without certain links or buses brings about easy scalability. Parallel working operators ensure low latencies.

For means of hardware-in-the-loop simulations, a specialized dataflow processor (FLYSIG) is currently under way at C-Lab (Hardt and Kleinjohann, 1997). In order to increase runtime performance the FLYSIG processor is statically configured during an initialization phase. In order to find an appropriate quantity of operators, we had a close look at several typical mechatronic systems. The example of a vehicle model is shown in Figure 3. Note the frequent occurrence of trigonometric functions, such as sine, cosine, etc., that have to be supported efficiently by the processor. 
Frequency of basic operations for a vehicle-model

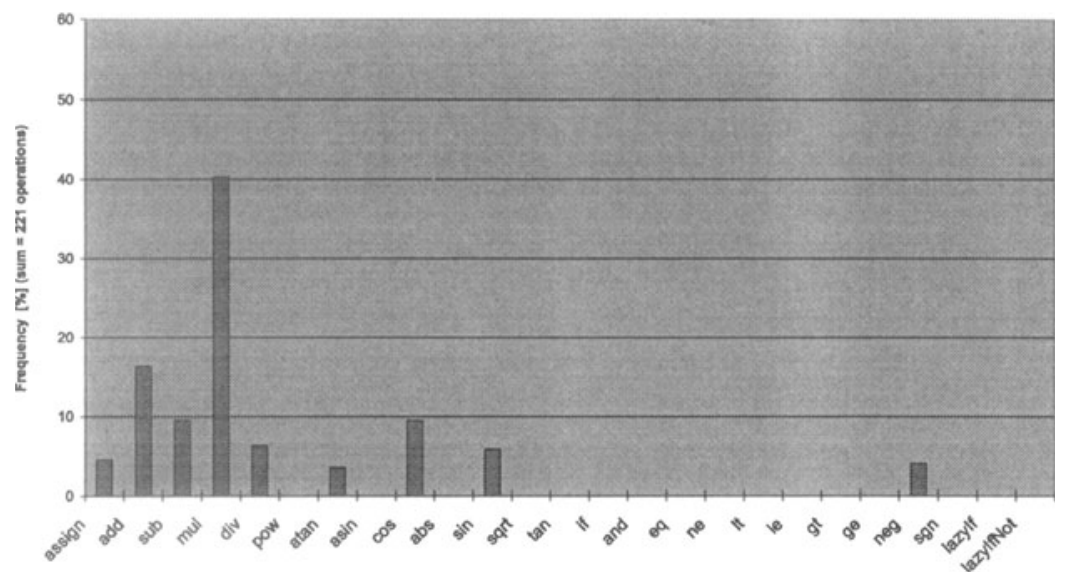

Figure 3. Frequencies of basic operations for a vehicle model.

\section{Example}

The approach presented here is applied to the design of an active vehicle suspension (Figure 4). The active vehicle suspension provides several advantages, such as improved riding comfort, enhanced driving safety and automatic leveling (Gillespie, 1992).

In addition to passive spring and damper elements, each suspension strut is equipped with a controlled hydraulic actuator. A main car controller computes the force setpoints for each local strut controller. The local setpoints are depending on the vertical position and velocity of the half-vehicle at each side of the car body. By this, less vertical acceleration and rolling of the car body can be achieved.

Therefore, we apply a hierarchical control concept. The local struts are typical Mechatronic Function Modules (MFMs) consisting of passive mechanical elements, actuators, sensors, and a local information-processing unit (controller).

For distributed simulation, it is important to partition the model appropriately. The physical topology of the model as well as its suitability for parallel computation have to be taken into account.

Models often include inherent parallel structures. The latter may help to find suitable partitions. Because of the complexity of mechatronic systems, it is necessary to apply the right granularity to partitioning. It is not useful to consider each and every equation of a model. We use the code classes of the basic systems as the smallest units.

The interdependencies of the code classes $N D, D$, and $S$ of each basic model component make up an acyclic directed graph. The tool DSCBIND is able to generate such a graph automatically from the $D S C$ model-description language (Figure 5). 


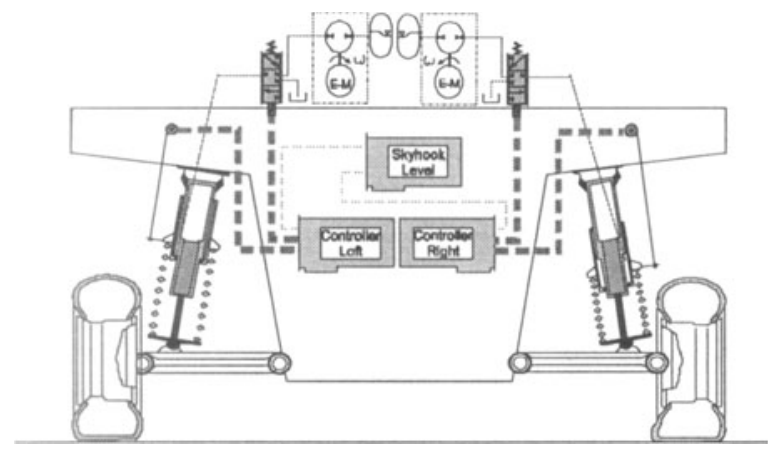

Figure 4. Half-vehicle with active suspension.

\subsection{Code Structure of the Model of the Half-vehicle}

The two struts are coupled to the rigid body (RigidBody_ND, RigidBody_S) and the main vehicle controllers (Level_D, Skyhook_D) by $D$ code of the spring and damper basic systems (SpringLeft_D, SpringRight_D, DamperLeft_D, and DamperRight_D). The code relating to the struts can be entirely executed in parallel.

This inherent parallel structure leads directly to one partition of the main controllers comprising the rigid body and one partition per strut comprising the local controllers. These partitions take into account the MFMs of the model and allow efficient parallel computing.

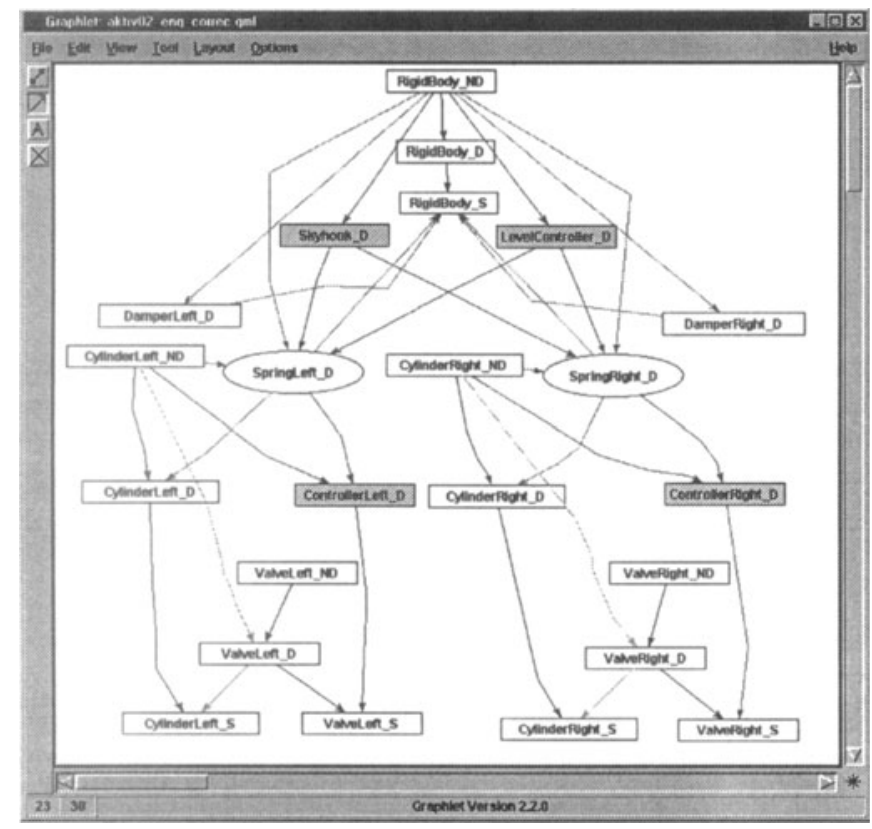

Figure 5. Code graph of the active suspension model of the half-vehicle. 


\subsection{Clustering}

When the partitions are transferred to the cluster concept, the clusters $A, B$, and $C$ will arise (Figure 6). For this clustering, there are the following demands on communication: cluster $B$ has to send the states of the rigid body and the controller setpoints to either of the struts (clusters $A$ and $C$ ). Clusters $A$ and $C$ send back the strut forces to cluster $B$. These connections are established as black-box couplings.

To gain exact numeric results all clusters should use the same rates for computation and communication. Additionally, the same solver for the differential equations should be used. Alteration of local solvers, of computation and/or communication rates (multirate) will have a large effect on the numerical accuracy.

In this example the eigenfrequencies of the rigid body $(\approx 1 \mathrm{~Hz})$ in cluster $B$ are more than ten times lower than the eigenfrequencies (wheel eigenfrequencies) of the struts in clusters $A$ and $C$. Due to this, a distributed multirate simulation is also compared with the standard simulation results.

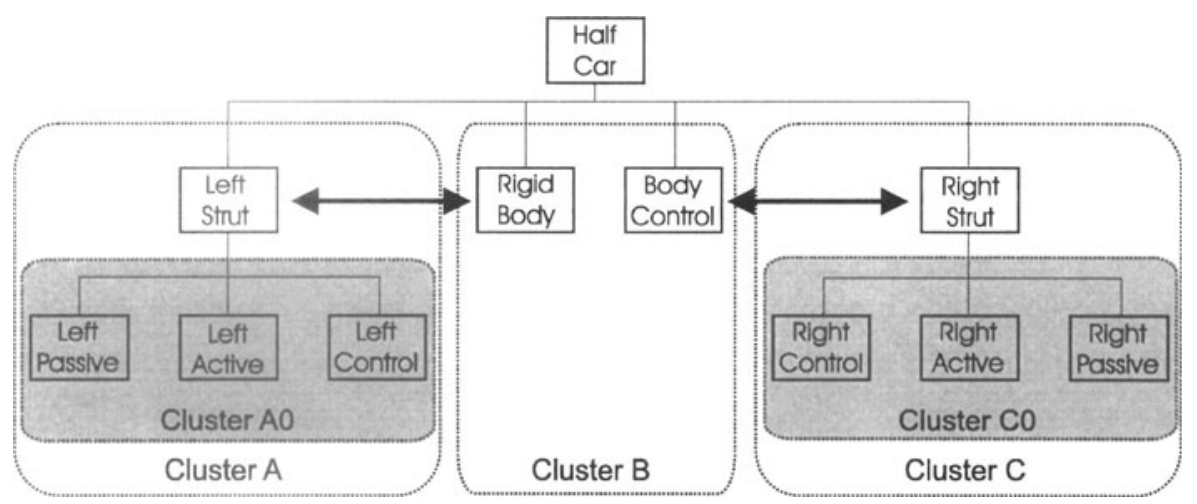

Figure 6. Clustering of the half-vehicle model for distributed simulation.

\subsection{Scheduling for T800 Transputer Hardware}

Prediction of the worst-case execution time (WCET) is very useful to evaluate the efficiency of parallel execution for a given clustering. It will make possible to do optimizations before implementing the application on the target platform. For the T800 transputer the tool DSCCOST is able to estimate the execution times of every basic system of a model. Caching or pipelining effects of processors are not taken into consideration. As a model for the asynchronous communication the $\log P$ model is used (Culler et al., 1993).

In addition to the clustering, the computational load of the basic systems and the $\log P$ parameters, the scheduling of the code blocks can also be computed and the WCET of one evaluation step predicted. Figure 7 shows the scheduling of our example. Computation, idling times, "send" overheads, "receive" overheads, and latencies are graphically represented.

For this example, a parallel WCET of 812 microseconds in comparison with sequential 1343 microseconds is predicted (Figure 7). 


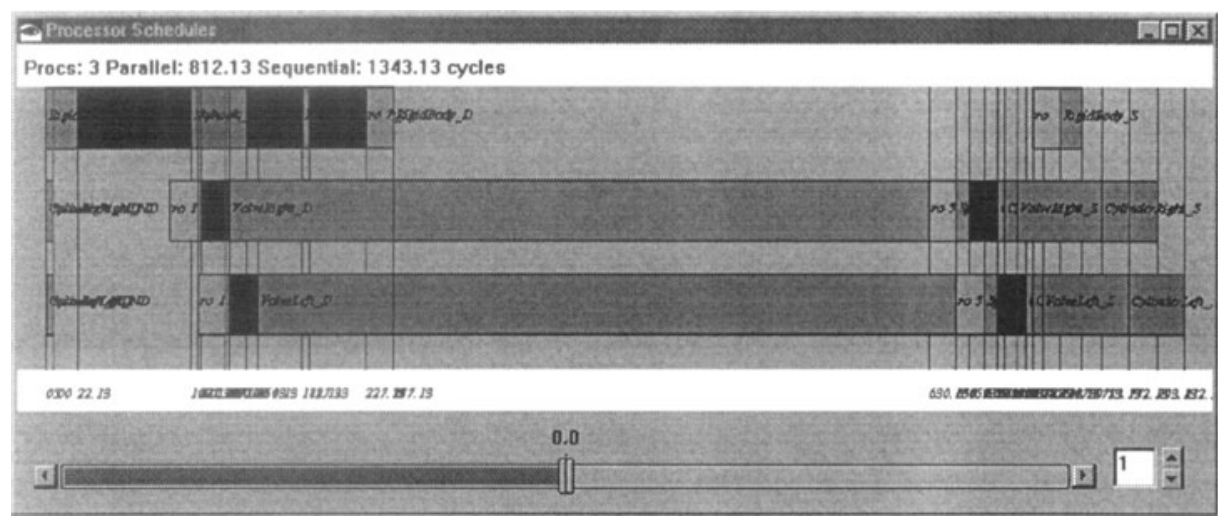

Figure 7. Graphical view of the code-block scheduling for three T800 transputers.

\subsection{Simulation Results}

The model described can be simulated by means of the runtime platform IPANEMA (Honekamp, 1998; Stolpe and Zanella, 1998)). Distributed off-line simulations on WindowsNT or UNIX hosts as well as on-line simulations on a transputer network are supported.

As an external excitation to the model, we chose a harmonic lateral body acceleration. It serves to examine cornering effects on the vehicle body. The vertical position on the outer body side and the roll angle are compared in active and in passive mode.

To simulate the model a Runge-Kutta solver of $4^{\text {th }}$ order with constant stepsize was used. The simulation stepsize was 1 millisecond. Four communication steps per major integration step are necessary. Simulation with an Euler solver at a stepsize of 0.5 millisecond produces the same results in active and passive modes.

For the multirate simulation the solver type for the vehicle body is Euler and for the strut, Runge-Kutta of $4^{\text {th }}$ order. This brought about one communication step per major integration step. Inputs during intermediate integration steps of the RungeKutta solver are set to the major integration step inputs. The simulation stepsize has then to be reduced to 0.5 millisecond to bring about accurate results (Figure 8). This means about four times more calculations and the same number of communications as compared to the previous simulation.

The simulation results show the increased damping of the vehicle body during cornering in the active mode. The maximum roll angle in the active mode is less than half that of the passive mode for the chosen excitation. The model and the distributed simulation can easily be extended to a whole vehicle application with two or more axles. Then active damping for the pitch motion can also be taken into consideration. 

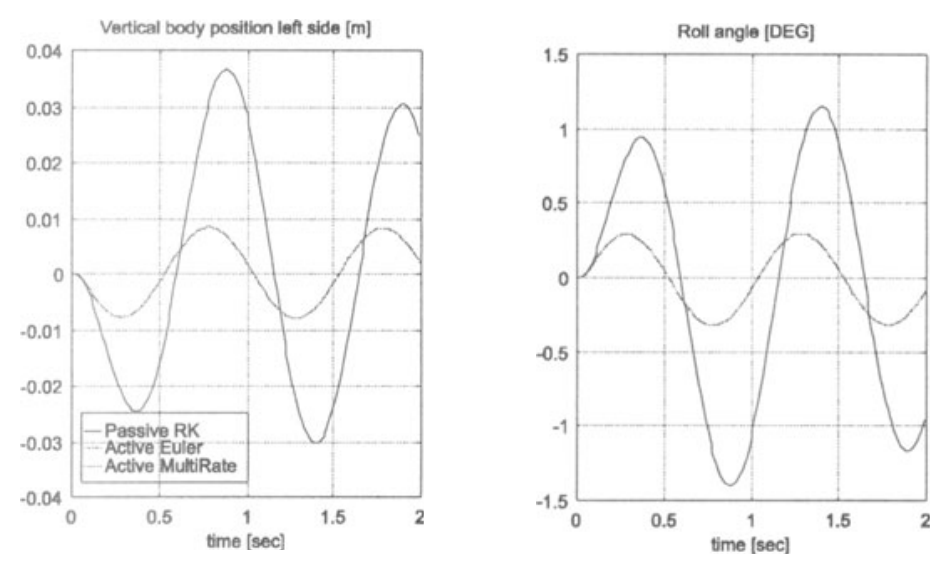

Figure 8. Simulation results for harmonic excitation with lateral acceleration of $1 \mathrm{~Hz}$ frequency and $1 \mathrm{~g}$ amplitude (cornering).

\section{References}

Altenbernd P. (1996). "On the False Path Problem in Hard Real-Time Programs." $8^{\text {th }}$ Euromicro Workshop on Real Time Systems (WRTS), L'Acquila, Italy.

Culler D., Karp R., Patterson D., Sahay A., Schauser K. E., Santos E., Subramonian R., Eicken T. (1993). "LogP: Towards a Realistic Model of Parallel Computation." $4^{\text {th }}$ ACM SIGPLAN Symposium on Principles and Practice of Parallel Programming, San Diego, CA.

Gillespie T. D. (1992). Fundamentals of Vehicle Dynamics, Society of Automotive Engineers, Inc., Warrendale, PA.

Hahn M., Richert J. (1994). "DSS - DSL - DSC. The Three Levels of a Model Description Language for Mechatronic Systems." Proceedings of the ICMA'94 "Mechatronics Spells Profitability", 777-794, Tampere, Finland.

Hardt W., Kleinjohann B. (1997). Eine asynchrone Datenflußarchitektur für eingebettete Systeme, Technical report 09/97, C-Lab, Paderborn, Germany.

Honekamp U. (1998). IPANEMA - Verteilte Echtzeit-Informationsverarbeitung in mechatronischen Systemen, Fortschr.-Ber. VDI, Reihe 20, Nr. 267. VDI-Verlag, Düsseldorf, Germany.

Honekamp U., Stolpe R., Naumann R., Lückel J. (1997). "Structuring Approach for Complex Mechatronic Systems." $30^{\text {th }}$ International Symposium on Automotive Technology \& Automation - Mechatronics/Automative Electronics, 165-172, Florence, Italy.

Homburg C., Naumann R. (1996). "LoDiT - Automatisches Partitionieren von mechatronischen Systemen für die verteilte Simulation." 10. Symposium Simulationstechnik, 279-284, Dresden, Germany.

Homburg C. (1998). "Mechatronic Processing Objects." 12. ASIM-Symposium Simulationstechnik, Posterband, ARGESIM Report Nr. 13, 53-60, Zurich, Switzerland.

Schröer J. (1994). Eine Modellbeschreibungssprache zur Unterstützung der Simulation und Optimierung von nichtlinearen und linearisierten hierarchischen Systemen. Fortschr.-Ber. VDI, Reihe 20, Nr. 128. VDI-Verlag, Düsseldorf, Germany.

Stolpe R.; Zanella M. (1998). "A Distributed Hardware-in-the-Loop Simulation Environment in Use on a Testbed of a Series Hybrid Drive." ESM'98 - 12th European Simulation Multiconference, Manchester, UK. 